\title{
Early Electrodiagnosis of the Ulnar Entrapment Neuropathy at the Elbow
}

\author{
Itaru Kimura, ${ }^{*}$ D.R. Ayyar and Susan M. Lippmann \\ Department of Neurology (D4-5), University of Miami \\ School of Medicine, Miami, Florida, USA
}

\begin{abstract}
Kimura, I., Ayyar, D.R. and Lippmann, S.M. Early Electrodiagnosis of the Ulnar Entrapment Neuropathy at the Elbow. Tohoku J. exp. Med., 1984. 142(2), 165-172 — Reliable, technically not demanding, electrophysiological tests make it possible to diagnose the ulnar entrapment neuropathy at the elbow. Ulnar nerve was stimulated supramaximally at the wrist, below and above the elbow, and in the upper arm, and the sensory nerve action potential (SNAP) and compound muscle action potential (CMAP) were recorded. Sensory and motor nerve conduction velocities (SNCVs, MNCVs) were calculated in each segment. In 12 of 64 symptomatic extremities of 44 patients with a presumptive diagnosis of the ulnar neuropathy at the elbow MNCVs of all segments were within normal limit and only SNCV across the elbow was significantly slowed $(p<0.001)$. In 25 extremities of the patients SNAP was not obtained. Evidence of denervation in the first dorsal interosseous and abductor digiti minimi muscles was detected in about $50 \%$ hands tested. This percentage was higher in the hands with absence of SNAP than those with presence of SNAPs. It would appear that measurement of SNCV across the elbow is a more sensitive parameter to detect abnormal conduction across the cubital tunnel in patients with the ulnar neuropathy._- ulnar nerve; entrapment neuropathy ; cubital tunnel syndrome; electrodiagnosis
\end{abstract}

An affection of the ulnar nerve is not difficult to diagnose but may be difficult to localize the lesion. To confirm the clinical diagnosis of the ulnar nerve lesion at the elbow it is customary to calculate motor nerve conduction velocity (MNCV) in each segment by stimulating the nerve at different sites (Simpson 1956; Eisen 1974 ; Miller 1979; Ring et al. 1980). As many of these patients with entrapment neuropathy present initially with sensory symptoms, it is felt that calculating sensory nerve conduction velocity (SNCV) in the entrapped segment may prove helpful in earlier diagnosis, especially when routine, more established methods fail to detect abnormal findings (Gilliatt and Thomas 1960; Payan 1969 ; Kimura and Ayyar 1983). Herein, we are describing a simple method to assess SNCV across the cubital tunnel in patients with a presumptive diagnosis of the ulnar neuropathy at the elbow and comparing these results to normal values

Received for publication, April 18, 1983.

* Present address: Department of Neurology, Institute of Brain Diseases, Tohoku University, Sendai 980, Japan. 
obtained in our laboratory.

\section{Materials And Methods}

All studies were carried out with the subjects supine with the arm $75^{\circ}$ to the trunk, the elbow extended and forearm supined. The skin temperature was checked over the forearm and maintained at $34^{\circ} \mathrm{C}$ or higher.

A TECA electromyograph (TE-4) was used to stimulate the nerves and to record responses and electromyogram (EMG). The sensory nerve action potentials (SNAPs) and compound muscle action potentials (CMAPs) were recorded on photosensitive paper for measurement.

Sensory nerve conduction studies. The high and low frequency filters of the EMG amplifier were set at $3.2 \mathrm{kHz}$ and $32 \mathrm{~Hz}$, respectively. The ulnar nerve was stimulated with surface stimulating electrodes (TECA 9523) at the wrist, below and above the elbow, and in the upper arm. SNAPs were recorded with digital ring electrodes (TECA 6032) placed around the little finger. SNCV of each segment (from wrist to below-elbow, across the elbow, and from above-elbow to upper arm) was calculated dividing distance between two different stimulating sites by difference of latencies measured from stimulating artifact to the onset of the response in each site (Kimura, Ayyar and McVeety 1983). The amplitude of the SNAPs was measured from peak to peak.

Motor nerve conduction studies. The high and low frequency filters were set at $8 \mathrm{kHz}$ and $8 \mathrm{~Hz}$, respectively. CMAPs were recorded with surface electrodes (TECA 6080) placed over the muscle belly of the abductor digiti minimi muscle and its tendon. The nerve was stimulated at four different sites in the same manner. MNCV of each segment was calculated. The amplitude of CMAPs was measured from the baseline to the negative peak of response.

Electromyography (EMG). Needle EMG was recorded from the first dorsal interosseous (FDI), abductor digiti minimi (ADM), flexor digitorum profundus of the third and fourth fingers (FDP) and flexor carpi ulnaris (FCU) muscles with concentric needle electrode (DISA 13L50). The high and low frequency filters were set at $8 \mathrm{kHz}$ and $8 \mathrm{~Hz}$, respectively.

Patients. Lesions of 64 ulnar nerves, 35 right and 29 left, were studied in 44 patients, 30 men and 14 women with frank clinical signs and symptoms suggestive of the ulnar neuropathy at the elbow. Patients were excluded from our series who had history of trauma, clinical or roentgenographic evidence of joint deformity or diseases that predisposed to peripheral neuropathy. Their ages ranged from 18 to 64 (mean 41.6 years). Of 44 patients, $20(45.5 \%)$ had clinical signs and symptoms of bilateral involvement.

Normal values of the SNCVs and MNCVs of all segment were measured in 32 normal extremities of 25 healthy volunteers, 15 men and 10 women. Their ages ranged from 20 to 66 (mean 40.8 years).

\section{Results}

In 12 of 64 symptomatic extremities tested MNCVs of all segments were within normal limit and only SNCN across the elbow was significantly slowed when compared to SNCVs of the forearm and of the arm segments $(p<0.001)$. Twenty-seven symptomatic extremities showed significant slowing of both SNCV and MNCV across the elbow $(p<0.001)$. In 25 symptomatic extremities SNAP was not obtained. In 32 normal controls MNCVs of all segments were normal, while SNCV across the elbow was slightly slowed when compared to SNCV of the forearm segment $(p .<0.01)$.

These results were classified into following four groups : normal group (G-1) ; 
extremities with normal MNCV and abnormal SNCV group (G-2); extremities with abnormal SNCV and MNCV group (G-3); and extremities with abnormal MNCV and absence of SNAP group $(\mathrm{G}-4)$. Percentage sensory nerve conduction velocity (\% SNCV) across the elbow was calculated with SNCV of the forearm segment being taken as $100 \%$. And percentage motor nerve conduction velocity $(\% \mathrm{MNCV})$ across the elbow was calculated in the same manner. These results are summarized in Tables 1 and 2. In Fig. 1 SNCVs and MNCVs of all segments in the 64 symptomatic extremities and 32 normal controls are represented.

Averaged amplitude of SNCVs in each group is represented in Fig. 2. Averaged amplitude of CMAPs in each group is shown in Fig. 3.

EMG was carried out in 60 symptomatic extremities of patients. Fibrillation potentials, positive sharp waves or both in more than two sites of the muscle outside the endplate zone were recorded as often in the FDI muscle as in the ADM muscle. The incidence of denervation in these muscles was higher in the hands with absence of SNAP than in those with presence of SNAPs. No evidence of denervation was noted in any muscles of patients with normal MNCV in each segment. The incidences of of denervation in the ADM, FDI, FDP and FCU muscles of patients of each group are shown in Table 3 .

\section{Discussion}

The main clinical features of the ulnar entrapment neuropathy at the elbow are: 1) no evidence of joint deformity or prior elbow trauma; 2) frequent occurrence of bilateral symptoms and signs of ulnar neuropathy; 3) a taut, palpably enlarged nerve in the ulnar groove ; 4) electrophysiological localization

TABLE 1. Ulnar nerve sensory conduction velocity (SNCV) of each segment in normal controls and patients with ulnar neuropathy at the elbow

\begin{tabular}{|c|c|c|c|c|c|}
\hline & & \multicolumn{3}{|c|}{$\mathrm{SNCV}(\mathrm{m} / \mathrm{sec})$} & \multirow{2}{*}{$\begin{array}{c}\% \text { SNCV } \\
\text { across-elbow }\end{array}$} \\
\hline & & Forearm & Across-elbow & Arm & \\
\hline Group 1 & $\begin{array}{c}\text { Normal } \\
\text { controls } \\
n=32\end{array}$ & $62.0 \pm 5.4^{*}$ & $\begin{array}{rl} & 57.3 \pm 5.5 \\
{ }^{*} & p<0.01 \\
* * & p<0.2\end{array}$ & $60.1 \pm 4.6^{* *}$ & $91.7 \pm 8.9 \%$ \\
\hline Group 2 & $\begin{array}{c}\text { With normal } \\
\text { MNCV } \\
n=12\end{array}$ & $60.4 \pm 6.2 \dagger$ & $\begin{array}{c}41.0 \pm 7.6 \\
\dagger p<0.001 \\
\dagger p<<0.001\end{array}$ & $64.0 \pm 6.6 \dagger \dagger$ & $\begin{array}{r}67.3 \pm 10.9 \% \\
\text { ๆ } p<0.001\end{array}$ \\
\hline Group 3 & $\begin{array}{c}\text { With } \\
\text { abnormal } \\
\text { MNCV } \\
n=27\end{array}$ & $61.8 \pm 3.1 \S$ & $\begin{array}{c}39.5 \pm 3.5 \\
\S p<0.001 \\
\S \S p<0.001\end{array}$ & $63.6 \pm 5.6 \S \S$ & $\begin{array}{c}63.5 \pm 6.3 \% \\
\text { ๆ } p<0.001\end{array}$ \\
\hline
\end{tabular}

Values represent mean \pm S.D.

$\%$ SNCV across-elbow was calculated as SNCV of the forearm segment was taken as

$n=$ number of extremities tested. 
TABLE 2. Ulnar motor nerve conduction velocity (MNCV) of each segment in normal controls and patients with ulnar neuropathy at the elbow

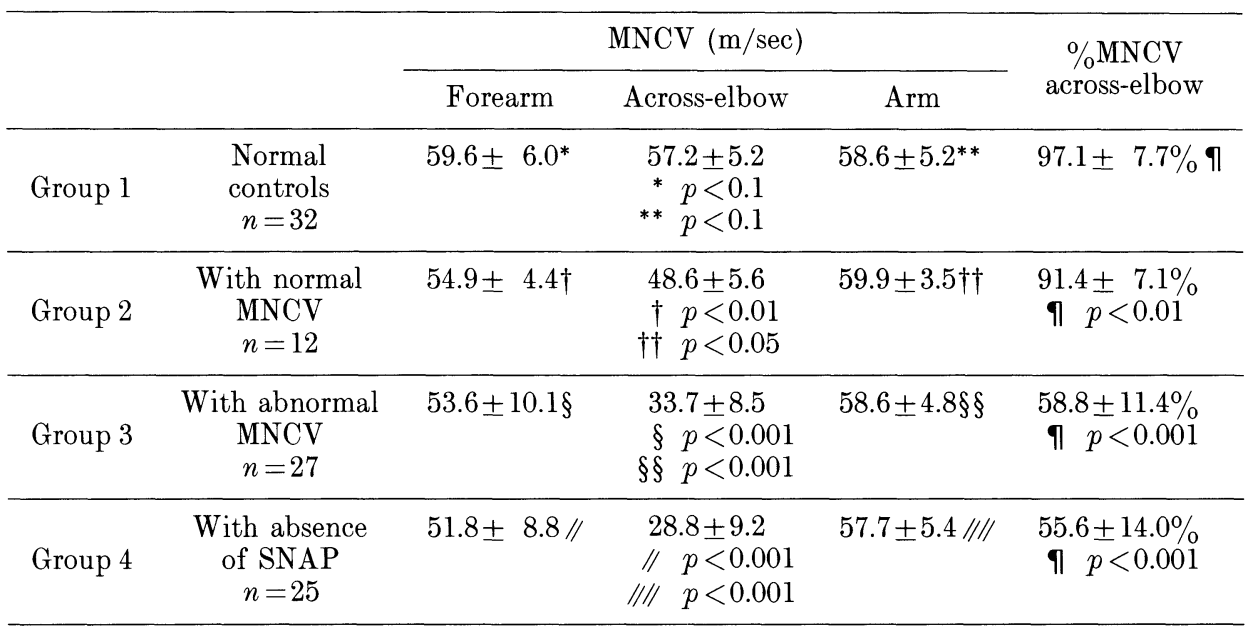

Values represent mean \pm S.D.

$\%$ MNCV across-elbow was calculated as MNCV of the forearm was taken as $100 \%$. $n=$ number of extremities tested.

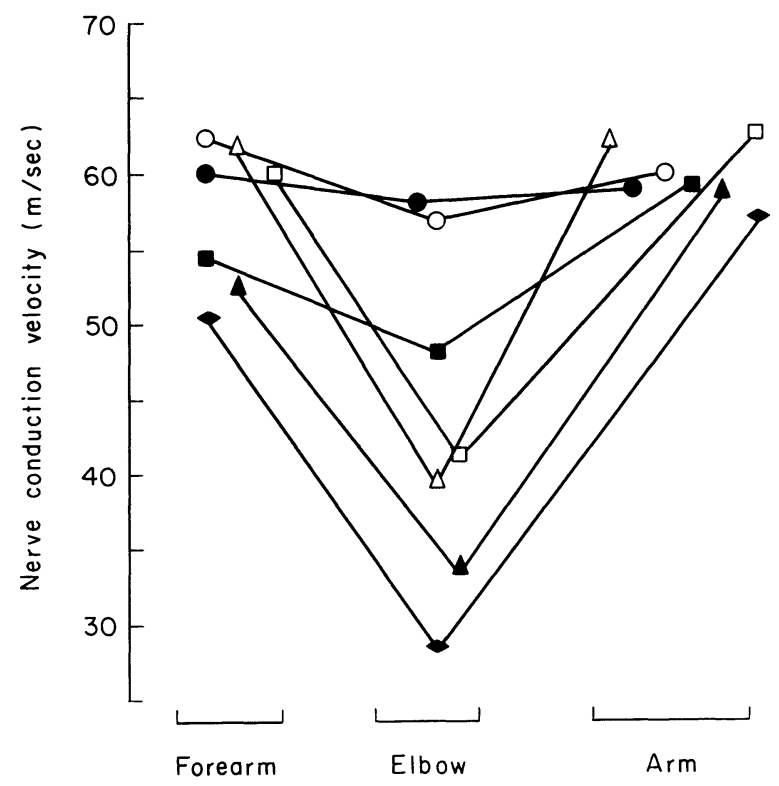

Fig. 1. Mean SNCV and MNCV of each segment in normal controls and patients with ulnar neuropathy at the elbow. $\bigcirc, \bullet=$ SNCV and MNCV in normal controls (Group 1); $\square, \boldsymbol{a}=\mathrm{SNCV}$ and MNCV in patients (Group 2); $\triangle$, $\Delta=\mathrm{SNCV}$ and MNCV in patients (Group 3) and $\boldsymbol{\Delta}=\mathrm{MNCV}$ in patients (Group 4). 


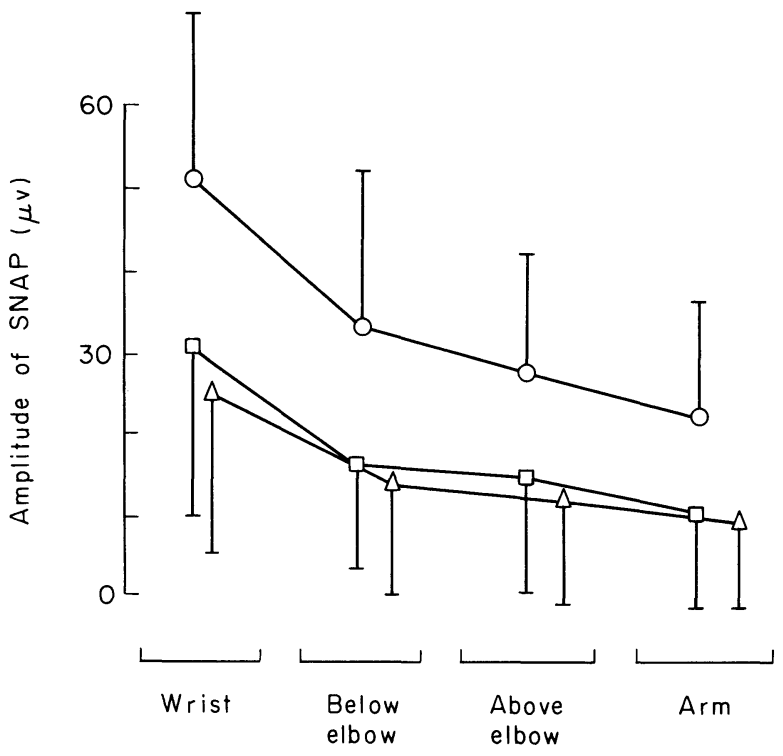

Fig. 2. Amplitude of SNAPs in each site of nerve stimulation. Values represent mean \pm s.D. $O=$ normal controls (Group 1); $\square=$ patients (Group 2) and $\Delta=$ patients (Group 3).

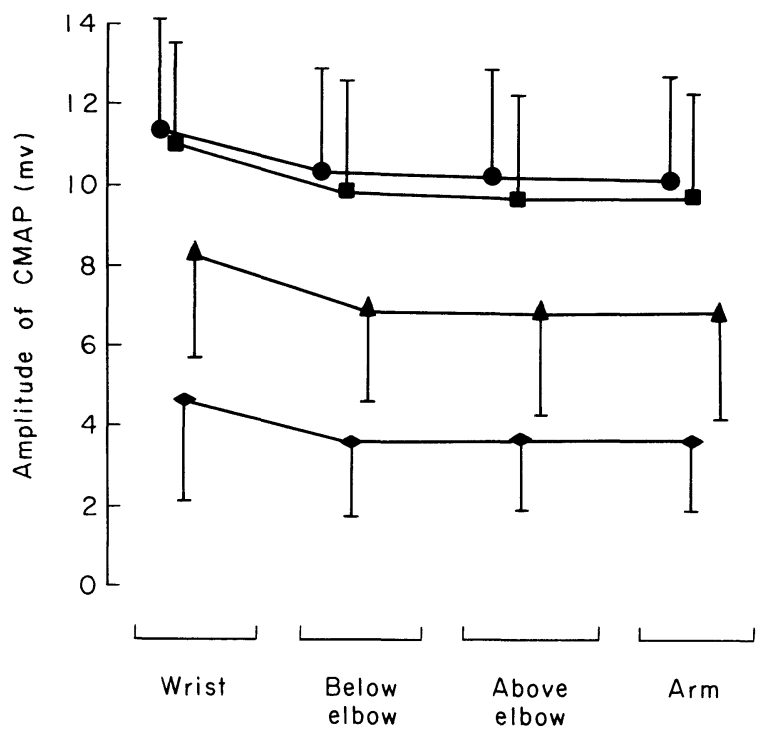

Fig. 3. Amplitude of hypothenar CMAPs in each site of nerve stimulation. Values represented mean \pm s.D. $\quad=$ normal controls (Group 1); $\mathbf{a}=$ patients (Group 2); $\boldsymbol{\Delta}=$ patients (Group 3) and $\boldsymbol{\nu}=$ patients (Group 4). 
TABLE 3. Incidence of denervation in the muscles supplied by the ulnar nerve in patients with ulnar neuropathy at the elbow

\begin{tabular}{|c|c|c|c|c|c|}
\hline & & $\mathrm{ADM}$ & FDI & FDP & $\mathrm{FCU}$ \\
\hline Group 2 & $\begin{array}{l}\text { With normal } \\
\text { MNCVs } \\
n=10\end{array}$ & $0 \%$ & $0 \%$ & $0 \%$ & $0 \%$ \\
\hline Group 3 & $\begin{array}{l}\text { With abnormal } \\
\text { MNCVs } \\
n=25\end{array}$ & $40 \%$ & $44 \%$ & $16 \%$ & $4 \%$ \\
\hline Group 4 & $\begin{array}{c}\text { With absence } \\
\text { of SNAP } \\
n=25\end{array}$ & $76 \%$ & $80 \%$ & $32 \%$ & $8 \%$ \\
\hline Total & $n=60$ & $50 \%$ & $53.3 \%$ & $20 \%$ & $6.7 \%$ \\
\hline
\end{tabular}

ADM, abductor digiti minimi ; FDI, first dorsal interosseous ; FDP, flexor digitorum profundus of the third and fourth fingers; FCU, flexor carpi ulnaris muscles; MNCVs, motor nerve conduction velocities and SNAP, sensory nerve action potential.

$n=$ numbers of extremities tested.

to the cubital tunnel ; and 5) operative findings of a swollen, taut, hyperemic nerve, distally limited by the proximal border of the aponeurosis jointing the two heads of the flexor carpi ulnaris muscle (Miller 1979). All patients of our series satisfied all of these criteria except 5) operative findings.

In nine of 15 ulnar nerves of Miller's series (1979), abnormal conduction was localized to the level of the cubital tunnel $(1.5$ to $3.5 \mathrm{~cm}$ distal to the medial epicondyle). Brown et al. (1980) studied with utilizing intraoperative nerve stimulation at the time of surgical transposition and found that most common conduction abnormalities were extending over several centimeters but maximum just proximal to, at the level of, or within one to two centimeters distal to the medial epicondyle. Precise localization has not determined in operative findings, however, it may be sufficient to determine precise localization electrophysiologically that nerve is stimulated at least five centimeters distal and five centimeters proximal to the medial epicondyle.

Presence of the purely sensory lesions that only sensory fibers show electrophysiological abnormalities and that motor fibers are not involved alone leads us to the concept that sensory fibers are the first to be damaged in patients with the ulnar neuropathy at the elbow. The same findings have been observed in other entrapment neuropathy (Kimura and Ayyar 1983). Payan (1969) described abnormal ulnar SNCV in the clinically normal arms of three patients with idiopathic unilateral neuropathy. In our series, two non-symptomatic extremities showed abnormal SNCV across the elbow without any abnormalities in MNCVs. Even in normal extremities of healthy volunteers, slight slowing of SNCV across the elbow, though it was below 10 percent slowing, was noted in our 
studies.

These results form a conception that every non-symptomatic extremity may be exposed to great risk of the ulnar entrapment lesion at the elbow. Only a few external factors such as constant pressure and repeated minimal trauma at the elbow as well as internal factors such as a subtle change in the anatomical dimensions of the cubital tunnel (bounded by the medial epicondyle, the olecranon and fibrous arch connecting them) and thickening of the nerve could manifest these subclinical signs and symptoms.

For earlier detection of abnormal conduction of the ulnar nerve at the cubital tunnel, measurement of SNCV across the elbow and representation of percentage slowing of SNCV across the elbow as SNCV of the forearm segment is taken as $100 \%$ may be a more sensitive and reliable parameter than other more established parameters. Early detection of abnormality is regarded as essential if protective measures could prevent nerve damage or if surgical interventions such as simple decompression, anterior nerve transposition and medial epicondylectomy (Eisen and Danon 1980; Miller and Hummel 1980; Craven and Green 1980) are performed while damage is still reversible.

The amplitude of the hypothenar CMAPs and ulnar SNAPs recorded with above-elbow nerve stimulation was smaller than the amplitude of the CMAPs and SNAPs with below-elbow stimulation. Miller (1979) described that precise localization of the conduction block depended on an abrupt amplitude change at the cubital tunnel using an inching technique. Our results failed to demonstrate significant change in amplitude of the SNAPs or the CMAPs at the cubital tunnel.

Payan (1969) reported incidence of denervation was 57.1\% in the hypothenar muscles and was $56.5 \%$ in the FDI muscle. These values are very close to our results of $50.0 \%$ and $53.3 \%$ in the $\mathrm{ADM}$ and FDI muscles, respectively. These percentages were higher in the hands with absence of ulnar SNAP than in those with presence of SNAPs. Evidence of denervation was also detected in the FDP and FCU muscles in our series.

Clinically the FCU muscle is usually spared because the nerve branch to this muscle exits the nerve proximal to the cubital tunnel. The nerve branch to the FDP exits the main nerve trunk distal to the aponeurosis, and finger flexion of the fourth finger is usually involved. Presence of evidence of denervation in the FCU muscle, although its percentage was only eight percent in the symptomatic hands with absence of ulnar SNAP, may suggest that damage of the nerve could be extending proximally to the nerve branch to FCU muscle in such severely involved cases with ulnar entrapment neuropathy at the elbow. 


\section{References}

1) Brown, W.F., Yates, S.K. \& Ferguson, G.G. (1980) Cubital tunnel syndrome and ulnar neuropathy. Ann. Neurol., 7, 289-290.

2) Craven, P.R., Jr. \& Green, D.P. (1980) Cubital tunnel syndrome. Treatment by medial epicondylectomy. J. Bone Joint Surg., 62, 986-989.

3) Eisen, A. (1974) Eary diagnosis of ulnar nerve palsy. An electrophysiologic study. Neurology (Minneap.), 24, 256-262.

4) Eisen, A. \& Danon, J. (1974) The mild cubital tunnel syndrome. Its natural history and indications for surgical intervention. Neurology (Minneap.), 24, 608-613.

5) Gilliatt, R.W. \& Thomas, P.K. (1960) Changes in nerve conduction with ulnar lesions at the elbow. J. Neurol. Neurosurg. Psychiat., 23, 312-320.

6) Kimura, I. \& Ayyar, D.R. (1983) The carpal tunnel syndrome. Electrophysiologic aspects in 639 cases. Electromyograph. clin. Neurophysiol. (in press).

7) Kimura, I., Ayyar, D.R. \& McVeety, J.C. (1983) Saphenous nerve conduction in healthy subjects. Tohoku J. exp. Med., 140, 67-71.

8) Miller, R.G. (1979) The cubital tunnel syndrome: Diagnosis and precise localization. Ann. Neurol., 6, 56-59.

9) Miller, R.G. \& Hummel, E.E. (1980) The cubital tunnel syndrome: Treatment with simple decompression. Ann. Neurol., 7, 567-569.

10) Payan, J. (1969) Electrophysiological localization of ulnar nerve lesions. J. Neurol. Neurosurg. Psychiat., 32, 208-220.

11) Ring, H., Costeff, H. \& Solzi, P. (1980) Criteria for preclinical diagnosis of the cubital tunnel syndrome. Electromyograph. clin. Neurophysiol., 19, 459-466.

12) Simpson, J.A. (1956) Electric signs in the diagnosis of carpal tunnel and related syndromes. J. Neurol. Neurosurg. Psychiat., 19, 275-280. 